\title{
Quark and hadron degrees of freedom in the Roper resonance electroproduction
}

\author{
I.T. Obukhovsky*, ${ }^{*}$ A. Faessler, ${ }^{b}$ D.K Fedorov, ${ }^{a}$ Th. Gutsche ${ }^{b}$ and V.E. Lyubovitskij ${ }^{b}$ \\ ${ }^{a}$ Institute of Nuclear Physics, Moscow State University, 119991 Moscow, Russia \\ ${ }^{b}$ Institute for Theoretical Physics, Tuebingen University \\ Auf der Morgenstelle 14, D-72076 Tuebingen, Germany \\ E-mail: bbukh@nucl-th.sinp.msu.ru
}

\begin{abstract}
The Roper resonance is considered as a mixed state of a three-quark core configuration and a hadron molecular component $N+\sigma$. Based on this ansatz we study electroproduction of the Roper resonance. The strong and electromagnetic couplings induced by the quark core are calculated in the ${ }^{3} P_{0}$ model. The contribution of the vector meson cloud to the electromagnetic transition is given in the framework of the VMD model. Results are compared with the recent JLab electroproduction data.
\end{abstract}

XXI International Baldin Seminar on High Energy Physics Problems,

September 10-15, 2012

JINR, Dubna, Russia

\footnotetext{
*Speaker.

${ }^{\dagger}$ Supported by the DFG grant FA67/40-1
} 


\section{Introduction}

The structure issue of the lowest lying nucleon resonance $N(1440)$ with $J^{P}=\frac{1}{2}^{+}$(the Roper resonance $P_{11}$ or simply $R$ ) has been a long standing problem of hadron physics. The simplest description of the Roper consists of the three-quark $(3 q)$ configuration $s p^{2}[3]_{X}$, i.e. the first $(2 S)$ radial excitation of the nucleon ground state $s^{3}[3]_{X}$, but it fails to explain either the large decay width $\Gamma_{R} \simeq 300 \mathrm{MeV}$ or the branching ratios for the $\pi N$ (55-75\%) and $\sigma N(5-20 \%)$ decay channels [1. 2]. Evaluation of these values in the framework of the CQM is often based on the elementary emission model (EEM) with single-particle quark-meson (or quark-gamma) couplings $q q \pi$, $q q \sigma, q q \gamma$, etc.. The calculation of decay widths (or of the electroproduction cross section at small virtuality of the photon with $Q^{2} \simeq 0$ ) results in anomalous small values. These underestimates can especially be traced to the strict requirement of orthogonality for the ground $(0 S)$ and excited state (2S) radial wave functions of the $N$ - and $R$ states belonging to the quark configurations with the same spin-isospin $(S=1 / 2, T=1 / 2)$ and symmetry $\left([3]_{S T}[3]_{X}\right)$ quantum numbers. To overcome this discrepancy it is suggested that either the Roper is not an ordinary $3 q$ state or the "true" transition operators have a more complicated form than the single-particle operators used in calculations. Recently the coupled channel EBAC-DCC approach successfully explained low P11(1440) mass as a result of a substantial shift of its bare quark core mass caused by meson-baryon dressing [3]

On the experimental side there has been noticeable progress in the experimental study of the Roper resonance in the last decade. CLAS data on unpolarized cross sections for major meson electroproduction channels $N \pi$ and $N \pi \pi$, complemented by the data on beam/beam-target asymmetries for $\mathrm{N} \pi$ electroproduction [4, 5] enhanced considerably our capabilities for extraction of P11(1440) electrocouplings in a wide range of photon virtualities $Q^{2}<5.0 \mathrm{GeV}^{2}$. The Roper resonance has also been studied a combined analysis of pion- and photo-induced reactions made by CB-ELSA and the A2-TAPS Collaborations [2]. These recent data present new possibilities for the study of the lightest baryon resonances.

Several models for the description of the Roper resonance electroexcitation were proposed during the last three decade (e.g., see the review [6]). Now model predictions can be compared with

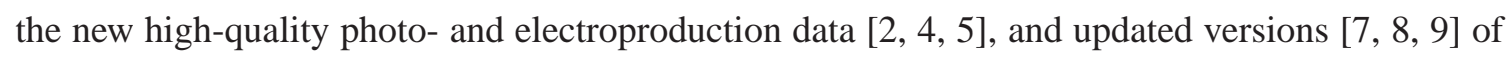
the most realistic models give a good description of the data at intermediate values of $1.5 \lesssim Q^{2} \lesssim$ $4 \mathrm{GeV}^{2}$. However, in the "soft" region, i.e. at low values of $Q^{2}\left(0 \leq Q^{2} \lesssim 1-1.5 \mathrm{GeV}^{2}\right)$, the data differ qualitatively from the theoretical predictions: the experimental helicity amplitude $A_{1 / 2}$ changes sign at $Q^{2} \approx 0.5 \mathrm{GeV}^{2}$ and it is large and negative at the photon point $Q^{2}=0$. Theoretical predictions for $A_{1 / 2}$ are large and positive at $Q^{2} \approx 0.5 \mathrm{GeV}^{2}$ and quickly go to a small negative (or zero) value at the photon point.

For pion electroproduction in the resonance region $W \simeq m_{R}$ the behavior of the transverse helicity amplitude $A_{1 / 2}$ near the photon point $Q^{2} \gtrsim 0$ is most sensitive to the "soft" component of the resonance state, i.e. to the possible contribution of the meson cloud. Electroproduction amplitudes in this kinematical region are successfully analyzed in terms of the dynamical coupled channel model [10, 11], which is used at the Excited Baryon Analysis Center (EBAC) at JLab. The detailed description of the low- $Q^{2}$ CLAS $p \pi^{+} \pi^{-}$data [12] was obtained in Ref. [5] on the basis of JLab-Moscow (JM) model [13, 14] that incorporates all mechanisms seen in nine independent differential cross sections for the first time measured with the CLAS detector, and describes 

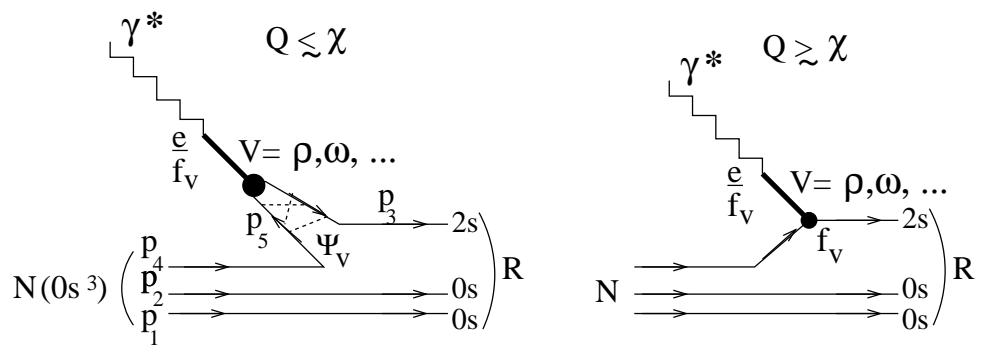

Figure 1: Diagrams of (a) "soft" (non-local) and (b) "hard" (local) coupling of vector mesons to the nucleon quark core.

$\mathrm{N} \pi \pi$ electroproduction amplitudes as a superpositon of six isobar channels: $\pi^{-} \Delta^{++}, \pi^{+} \Delta^{0}, \rho \mathrm{p}$, $\pi^{+} \mathrm{D} 13(1520), \pi^{+} \mathrm{F} 15(1685), \pi^{-} P 33^{++}(1620)$, and direct $2 \pi$ production mechanisms, when the final $\pi^{+} \pi^{-}$p state is created without formation of unstable hadrons in the intermediate states. The contribution of the meson (pion) cloud to the Roper resonance mass was recently calculated in Refs. [15, 16].

As a result, there are essentially two comprehensive theoretical approaches to the Roper electroproduction on the market. One of them (the coupled channel model of the meson cloud [5, 10, 11]) is only successful in the soft region $0 \leq Q^{2} \lesssim 1 \mathrm{GeV}^{2}$ and the other one (the light front (LF) three-quark model [7, 17] or the covariant quark spectator model [8]) is compatible with data in the hard region $1.5 \lesssim Q^{2} \lesssim 4 \mathrm{GeV}^{2}$. Theoretical analyses of the data on P11(1440) electrocouplings, carried out within the framework of quark models [18, 8, 9] and the EBAC-DCC coupled channel approach [11, 12] strongly suggest a combined contribution to this resonance structure from a core of three constituent quarks in the first radial excitation and meson-baryon dressing. The contribution of meson-baryon dressing is maximal at $Q^{2}<1 \mathrm{GeV}^{2}$. It may be well responsible for observed in this area of $Q^{2}$ difference between quark model expectations and the data [13, 14]. As $Q^{2}$ increases, the contribution of meson-baryon dressing becomes smaller, and at $Q^{2}>1.5 \mathrm{GeV}^{2}$ the data [13, 14] are consistent with a major contribution from a quark core.

Approaches which pretend to cover both regions of $Q^{2}$ were also suggested (see, e.g. Refs. [19] and [9]). In Ref. [19] a $3 q+\bar{q} q$ approach was suggested using the ${ }^{3} P_{0}$ model [20] and vector meson dominance (VMD) in combination with the EEM. In Ref. [9] a generalization of the Cloudy Bag Model (CBM) was used for the case of the open inelastic channels $\pi \Delta$ and $\sigma N$ in combination with a phenomenological strong background interaction. In such combined approaches two types of electromagnetic transition operators are used, the operator designed for the soft $Q^{2}$ region and one for hard values of $Q^{2}$. However, in the transition amplitude they are summed for any value of $Q^{2}$. For example, in the generalized ${ }^{3} P_{0}+$ EEM approach [19] the transition operator includes the sum of two vertices, schematically sketched in Figs. 17a and $b$.

In our recent work [21] we follow a more physical concept (see, e.g., Ref. [22] where the constituent quark and parton approaches to the $\gamma q q$ vertex are discussed in the context of the nucleon electromagnetic form factors). We can consider that the diagram in Fig. 19 represents the unknown large-distance physics described by a phenomenological model (the ${ }^{3} P_{0}$ model in our case), which is adjusted to low-energy data (i.e. meson-nucleon coupling constants $\pi N N, \rho N N$, magnetic moments and decay widths). In the hard $Q^{2}$-region these contributions become less important and an 
adequate description of the electromagnetic transition will be given by the diagram in Fig. 1 $1 \mathrm{~b}$. In this case the unknown short-range physics is encoded by adjusted parameters of a parton model. In the region of moderate values of $Q^{2}\left(1.5 \lesssim Q^{2} \lesssim 4 \mathrm{GeV}^{2}\right)$ we can consider the constituent quarks as partons and corresponding unknown short-range physics can be included in a few constituent quark parameters (such as quark form factors given by the intermediate vector mesons in the VMD model and scale parameters of quark configurations in the baryons). In this case it is not necessary to sum the contributions of the two diagrams in Fig. 1. Instead it would be desirable to use some mechanism for a smooth transition from one regime to the other. In our opinion such a mechanism can be described in general by a smooth transition from a typical hadron radius $b_{V} \approx 0.5 \mathrm{fm}$ of the vector meson in the CQM to a point-like vector meson $b_{V}=0$ corresponding to the quark-parton picture sketched in Fig. [1b. Here we use the approximation $b_{V}\left(Q^{2}\right)=b_{V}(0) e^{-Q^{2} / \chi^{2}}$, where $\chi \simeq 1-2$ $\mathrm{GeV}$ corresponds to the lowest characteristic value of $Q^{2}$ where the parton model phenomenology in deep inelastic ep scattering sets in.

Another important issue related to the Roper resonance is a possible combined structure of this state which implies a virtual hadron-hadron component (e.g. $\sigma N$ or/and $\pi \Delta$ ) [23] in addition to the radially excited three-quark structure. Here we consider an admixture of the hadronic molecular state $N+\sigma$ in an effective description of such a component. We also consider to what degree such a combined structure for the Roper is compatible with the new high-quality data of JLab.

\section{Composit structure of the Roper resonance}

We consider the Roper resonance $(R)$ as a superposition of the radially excited three-quark configuration $3 q^{*}$ and the hadron molecule component $N+\sigma$ as:

$$
|R\rangle=\cos \theta\left|3 q^{*}\right\rangle+\sin \theta|N+\sigma\rangle,
$$

where $\theta$ is the mixing angle between the $3 q^{*}$ and the hadronic component. In a first step we simplify the model by reducing it to two independent (decoupled) systems, $R_{1}=3 q^{*}$ and $R_{2}=N+\sigma$, and do not consider the full coupled channel problem. Moreover, we consider the dynamics of the $R_{1}$ component in the framework of the nonrelativistic ${ }^{3} P_{0}$ model (see, e.g. Refs. [20, 24]), while the dynamics of the $R_{2}$ component is considered in the framework of the hadronic molecular approach [25] which is manifestly Lorentz invariant.

The effective interaction term of the ${ }^{3} P_{0}$ model [24, 26] is set up as

$$
H_{q}^{\mathrm{eff}}=g_{q} \int d^{3} x \bar{\psi}_{q} \psi_{q}, \quad g_{q}=2 m_{q} \gamma,
$$

where $\gamma$ is dimensionless constant. Apart from some drawbacks, the ${ }^{3} P_{0}$ model [24] is a good phenomenological method for the evaluation of hadron transitions [27, 28] on the basis of the quark model starting from Eq. (2.2) with a single strength parameter $\gamma$. The interaction term (2.2) gives rise to Feynman amplitudes for the $\bar{q} q$-pair creation (annihilation)

$$
(2 \pi)^{3} \delta^{(3)}\left(\mathbf{p}_{4}+\mathbf{p}_{5}\right) i \mathscr{M}_{f i}^{\mathrm{eff}}=\left\langle q, \mathbf{p}_{4}, \mu_{4}\right|\left\langle\bar{q}, \mathbf{p}_{5}, \mu_{5}\left|i \int d^{3} x \mathscr{L}_{q}^{\mathrm{eff}}(x)\right| 0\right\rangle,
$$

which are used here for the calculation of meson-baryon couplings. The quark is labelled by its 3-momentum $\mathbf{p}_{4}$ and spin projection $\mu_{4}$ (for simplicity the isospin projection $t_{4}$ and the color are 
omitted), similarly for the antiquark. For the numbering of the quarks see Fig. 1. The corresponding non-relativistic interaction term $V_{q}^{\text {eff }}$ is defined as

$$
T_{f i}^{\mathrm{eff}}={ }_{n r}\left\langle q, \mathbf{p}_{4}, \mu_{4}\right|{ }_{n r}\left\langle\bar{q}, \mathbf{p}_{5}, \mu_{5}\left|V_{q}^{\mathrm{eff}}\right| 0\right\rangle \doteq \frac{1}{2 m_{q}} \mathscr{M}_{f i}^{\mathrm{eff}}
$$

that leads to the expression

$$
V_{q}^{\mathrm{eff}} \doteq \frac{g_{q}}{2 m_{q}}(-1)^{1-\mu_{5}-t_{5}}\left\langle\frac{1}{2}-\mu_{5}\left|\boldsymbol{\sigma} \cdot\left(\mathbf{p}_{4}-\mathbf{p}_{5}\right)\right| \frac{1}{2} \mu_{4}\right\rangle\left(\frac{1}{2}-t_{5} \mid \frac{1}{2} t_{4}\right)(2 \pi)^{3} \boldsymbol{\delta}^{(3)}\left(\mathbf{p}_{4}+\mathbf{p}_{5}\right),
$$

which is the nonrelativistic analogue of the $\bar{q} q$ pair creation (annihilation) operator.

The description of the hadronic $N+\sigma$ component of the Roper resonance is based on the compositeness condition [29, 30]. This condition implies that the renormalization constant of the hadron wave function is set equal to zero or that the hadron exists as a bound state of its constituents only. In the case of mixed states (as in the present situation where the Roper is a superposition of the $3 q^{*}$ and $N+\sigma$ components) the amplitude for the $N+\sigma$ component is defined by the parameter $\sin \theta$. The compositeness condition was originally applied to the study of the deuteron as a bound state of proton and neutron [29]. Then it was extensively used in low-energy hadron phenomenology as the master equation for the treatment of mesons and baryons as bound states of light and heavy constituent quarks (see e.g. Refs. [30, 31]). By constructing a phenomenological Lagrangian including the couplings of the bound state to its constituents and of the constituents to other particles in the possible decay channels we calculated hadronic-loop diagrams describing different decays of the molecular states (see details in [25]).

In the present case the $R_{2} \rightarrow N+\sigma$ coupling is fixed from the compositeness condition

$$
Z_{R}=1-\left.\Sigma_{N \sigma}^{\prime}(p)\right|_{\not p=m_{R}}=0,
$$

where $\Sigma_{N \sigma}(p)$ is the mass operator of the $N \sigma$ bound state, calculated with the use of the phenomenological Lagrangian

$$
\mathscr{L}_{R}^{\mathrm{str}}(x)=g_{R \sigma N} \bar{R}(x) \int d y \Phi_{R}\left(y^{2}\right) N\left(x+w_{\sigma N} y\right) \sigma\left(x-w_{N \sigma} y\right)+\text { H.c. },
$$

where $w_{i j}=m_{i} /\left(m_{i}+m_{j}\right)$. Here $\Phi_{R}\left(y^{2}\right)$ is the correlation function describing the distribution of $N \sigma$ inside $R$, which depends on the Jacobi coordinate $y$. Its Fourier transform used in the calculations has the form of a "modified" Gaussian, i.e. the Gaussian multiplied by a polynomial. In Euclidean space it may be written as

$$
\tilde{\Phi}_{R}\left(-k_{E}^{2}\right)=\left(1-\lambda \frac{k_{E}^{2}}{\Lambda_{M}^{2}}\right) \exp \left(-\frac{k_{E}^{2}}{\Lambda_{M}^{2}}\right)
$$

where $k_{E}$ is the Euclidean momentum. This present a kind of generalization of the nonrelativistic quark model wave function to the 4-dimensional case. But the relativistic parameters $\lambda$ and $\Lambda_{M}$ should differ from the corresponding nonrelativistic ones. Here $\Lambda_{M}$ is the molecular size parameter and $\lambda$ is a free parameter which should be fixed by the orthogonality condition, i.e. $\langle N \mid R\rangle=0$. 


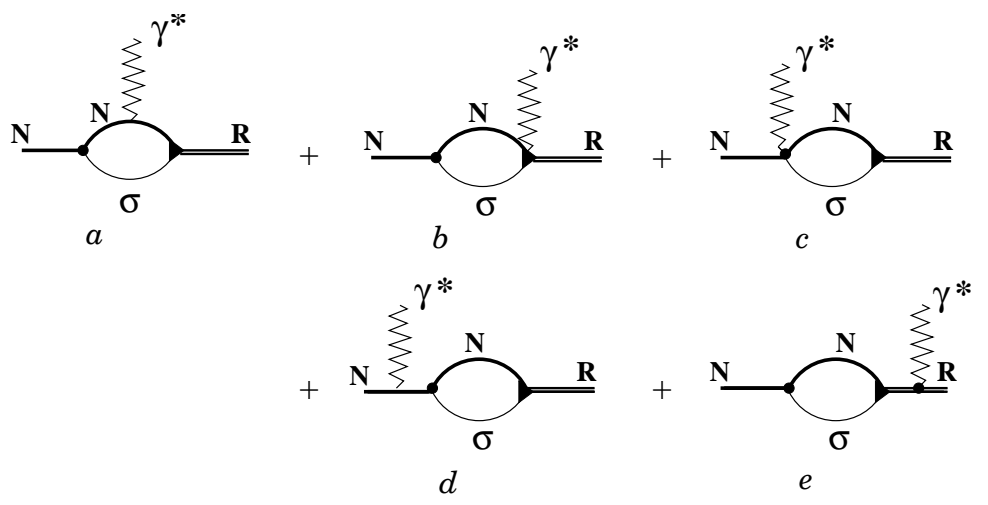

Figure 2: $N \sigma$ hadronic-loop diagrams contributing to the Roper electroproduction: the triangle diagram (a), the bubble diagrams (b) and (c), the pole diagrams (d) and (e).

\section{Roper electroproduction}

The diagrams which contribute to the Roper resonance electroproduction are shown in Fig. 1 and Fig. 3 . The transverse $(\lambda= \pm 1)$ and longitudinal $(\lambda=0)$ helicity amplitudes for electroproduction of the Roper resonance on the proton $\left(T_{z}=1 / 2\right)$ are defined by the matrix elements of the curent for $\lambda=+1$ and 0 respectively [32, 17, 8

$$
A_{1 / 2}=\sqrt{\frac{2 \pi \alpha}{q_{R}}}\left\langle R, \mathbf{0},+\frac{1}{2}\left|j_{q}^{\mu} \varepsilon_{\mu}^{(+)}\right| N,-\mathbf{q},-\frac{1}{2}\right\rangle, S_{1 / 2}=\sqrt{\frac{2 \pi \alpha}{q_{R}}}\left\langle R, \mathbf{0},+\frac{1}{2}\left|j_{q}^{\mu} \varepsilon_{\mu}^{(0)}\right| N,-\mathbf{q},+\frac{1}{2}\right\rangle \frac{|\mathbf{q}|}{Q}(3
$$

where $\alpha=1 / 137$ and we introduce $q_{R}=\frac{m_{R}^{2}-m_{N}^{2}}{2 m_{R}}$ for the threshold value of the photon 3-momentum for Roper electroproduction.

A. The contribution of the $3 q^{*}$ component to the hadronic current of the Roper electroproduction is generally given as

$$
J^{\mu}=\left\langle R\left|j_{q}^{\mu}\right| N\right\rangle \doteq\left\langle R, \mathbf{p}^{\prime}, S_{z}^{\prime}, T_{z}^{\prime}\left|j_{q}^{\mu}\right| N, \mathbf{p}, S_{z}, T_{z}\right\rangle .
$$

The current $j_{q}^{\mu}$ is derived by starting from the vector meson absorption amplitudes described in the ${ }^{3} P_{0}$ model

$$
T_{V+N \rightarrow R}^{q(\lambda)}=3_{n r}\left\langle R, \mathbf{0}, S_{z}^{\prime}, T_{z}^{\prime}\left|V_{q}^{e f f}\right| N,-\mathbf{q}, S_{z}, T_{z}\right\rangle\left|V, \mathbf{q}, \lambda_{V}, t_{V}\right\rangle_{n r}
$$

and use of the vector meson dominance (VMD) mechanism in the photon-quark coupling:

$$
e \varepsilon_{\mu}^{(\lambda)} J^{\mu}=\frac{e}{2} \sum_{V=\rho, \omega} \frac{\mathscr{M}_{V+N \rightarrow R}^{q(\lambda)}}{g_{V N N}} \frac{M_{V}^{2}}{Q^{2}+M_{V}^{2}} .
$$

The vector meson-nucleon coupling constant $g_{V N N}$ is calculated in the ${ }^{3} P_{0}$ model. $M_{V}$ is the vector meson mass approximated as $M_{V}=M_{\rho} \approx M_{\omega} ; \mathbf{p}, S_{z}, T_{z}\left(\mathbf{p}^{\prime}, S_{z}^{\prime}, T_{z}^{\prime}\right)$ and $\mathbf{q}, \lambda_{\rho}, t_{\rho}$ are the 3-momentum, spin and isospin projections of the nucleon (the Roper) and of the vector meson, respectively. For convenience we choose the photon momentum as $q^{\mu}=\left(q_{0}, 0,0,|\mathbf{q}|\right)$.

For the non-diagonal process $N+\gamma^{*} \rightarrow R$ in the limit $|\mathbf{q}|, q_{0} \rightarrow q_{R}$ (i.e. at the photon point) the matrix element of the transverse component $J^{\mu} \varepsilon_{\mu}^{(\lambda)}, \lambda= \pm 1$ of the current (3.2) defines 'the 
transition magnetic moment":

$$
\hat{\mu}_{N \rightarrow R}=-\frac{e}{2 m_{N}} \frac{\left(I+5 \tau_{z}\right)}{2} \frac{\sqrt{3}}{2} \exp \left[-\zeta\left(y_{0}\right) \mathbf{q}_{R}^{2} b^{2} / 6\right]\left[\frac{2 y_{0}^{2} / 3}{1+2 y_{0}^{2} / 3}-\left(\frac{1+y_{0}^{2}}{1+2 y_{0}^{2} / 3}\right)^{2} \frac{\mathbf{q}_{R}^{2} b^{2}}{9}\right]
$$

where $y_{0}=b_{V} / b, \zeta(y)=\frac{1+\frac{5}{6} y^{2}}{n}, n(y)=1+\frac{2}{3} y^{2}$. The quantity $\hat{\mu}_{N \rightarrow R}$ gives the value (apart from a kinematical factor $\left\langle\sigma_{+}\right\rangle \sqrt{q_{R} / 2}$ ) of the transverse helicity amplitude $A_{1 / 2}$ at the photon point.

The first term in the square brackets of the r.h.s. of Eq. (3.5) $Z_{V}=\frac{2 y_{0}^{2} / 3}{1+2 y_{0}^{2} / 3}$ is present because of the nonlocality of the $V q q$ interaction defined by Eq. (3.3). There the operator $V_{q}^{\text {eff }}$ leads to an insertion of the inner $\bar{q} q$ wave function of the vector meson into the $V q q$ vertex. The size of the nonlocal region is defined by the spatial scale of the meson wave function. For a point-like vector meson $\left(b_{V}=0\right)$ the value of $Z_{V}$ reduces to zero, and the matrix element for the transition $N+\gamma_{T}^{*} \rightarrow R$ reduces to the matrix element of the elementary-emission model (EEM) with a local $V q q$ vertex. The EEM matrix element vanishes in the limit $|\mathbf{q}| \rightarrow 0$, as it should because of the orthogonality of the spatial parts of the wave functions of $N$ and $R$.

Such behavior of the $A_{1 / 2}$ amplitude near the photon point $Q^{2}=0$ is characteristic of all the models which start from local $\gamma q q$ or $V q q$ vertices at high $Q^{2}$ and continue to use such interaction in the 'soft' region of small $Q^{2} \lesssim 6 / b_{V}^{2}$, where the e.-m. interaction is modified by the inner structure of vector mesons as $\bar{q} q$ bound states. As a result, in models with a local operator for the $\gamma q q$ (or $V q q$ ) interaction (see, e.g. the relativistic models [32, 17, 8]) the transverse helicity amplitude $A_{1 / 2}$ vanishes in the limit $Q^{2} \rightarrow 0$ [or it approaches a small value which is defined by the second term in the last line of Eq. (3.5) modified by relativistic corrections].

B. The hadronic $N \sigma$ loop diagrams contributing to the Roper electroexcitation are shown in Fig. 3. The $R N \sigma$ vertex is defined by the nonlocal Lagrangian $\mathscr{L}_{R}$ of Eq. (2.7). For the $N N \sigma$ vertex we use a similar nonlocal Lagrangian with the correlation function $\Phi_{N}\left(y^{2}\right)$

$$
\mathscr{L}_{N}=g_{N N \sigma} \sigma(x) \int d y \Phi_{N}\left(y^{2}\right) \bar{N}(x+y / 2) N(x-y / 2),
$$

where $g_{N N \sigma}$ is the $N N \sigma$ coupling constant, $\tilde{\Phi}_{N}\left(-k_{E}^{2}\right)=\exp \left(-\frac{k_{E}^{2}}{\Lambda_{N}^{2}}\right)$ is the Fourier transform of $\Phi_{N}\left(y^{2}\right)$ in Euclidean space with $\Lambda_{N}=0.7-1 \mathrm{GeV}$.

The electromagnetic interaction Lagrangian contains two pieces

$$
\mathscr{L}_{\text {int }}^{\mathrm{em}}=\mathscr{L}_{\text {int }}^{\mathrm{em}(1)}+\mathscr{L}_{\mathrm{int}}^{\mathrm{em}(2)}
$$

which are generated after the inclusion of photons. The first term $\mathscr{L}_{\text {int }}^{\mathrm{em}(1)}$ is standard and is obtained by minimal substitution in the free Lagrangian of the proton and charged Roper resonance:

$$
\partial^{\mu} B \rightarrow\left(\partial^{\mu}-i e_{B} A^{\mu}\right) B
$$

where $B$ stands for $p, R^{+}$and $e_{B}$ is the electric charge of the field $B$. The interaction Lagrangian $\mathscr{L}_{\text {int }}^{\mathrm{em}(1)}$ reads

$$
\mathscr{L}_{\text {int }}^{\mathrm{em}(1)}(x)=e_{B} \bar{B}(x) \not A B(x) .
$$

The second electromagnetic interaction term $\mathscr{L}_{\text {int }}^{\mathrm{em}(2)}$ is generated when the nonlocal Lagrangians (2.7) and (3.6) are gauged. The gauging proceeds in a way suggested and extensively used in Refs. [31, 
33, 34]. In order to guarantee local $U(1)$ gauge invariance of the strong interaction Lagrangian one multiplies each charged field in (2.7) and (3.6) with a gauge field exponential $e^{-i e_{B} I(y, x, P)}$. The exponent contains the term

$$
I(y, x, P)=\int_{x}^{y} d z_{\mu} A^{\mu}(z),
$$

where $P$ is the path of integration from $x$ to $y$. An expansion of the gauge exponential up to terms linear in $A^{\mu}$ leads to $\mathscr{L}_{\text {int }}^{\text {em }(2)}$. The full Lagrangian consistently generates the required matrix element of the electroexcitation amplitude which is linked to coming the hadronic molecular component of the Roper.

\section{Results and comparison with data}

In the calculation the helicity amplitudes $A_{1 / 2}$ and $S_{1 / 2}$ we use the free parameters typical for the CQM: $b=0.48 \mathrm{fm}, y_{0}=\frac{b_{V}}{b}=0.9, \chi^{2}=1.5 m_{N}^{2}, b_{R}=0.9444 b$. They were only fitted to the $A_{1 / 2}$ JLab data [ 4 , 可] without any additional adjustment to the $S_{1 / 2}$ data.

The set of parameters related to the molecular component includes the mixing parameter $\theta$, the scale parameters $\Lambda_{M}, \Lambda_{N}$ and the parameter $\lambda$ entering in the vertex function of the Roper. Further parameters linked to the $\sigma$ are the mass $M_{\sigma}$, the width $\Gamma_{\sigma}$ and the strong coupling constant $g_{\sigma N N}$. The parameters $\Lambda_{M} \approx \Lambda_{N} \approx 1 \mathrm{GeV}$ are approximately taken at the scale set by the light baryons. The parameter $\lambda$ is fixed through the orthogonality condition $\langle R \mid N\rangle=0$ (finally fitted at $\lambda=2.45$ ).
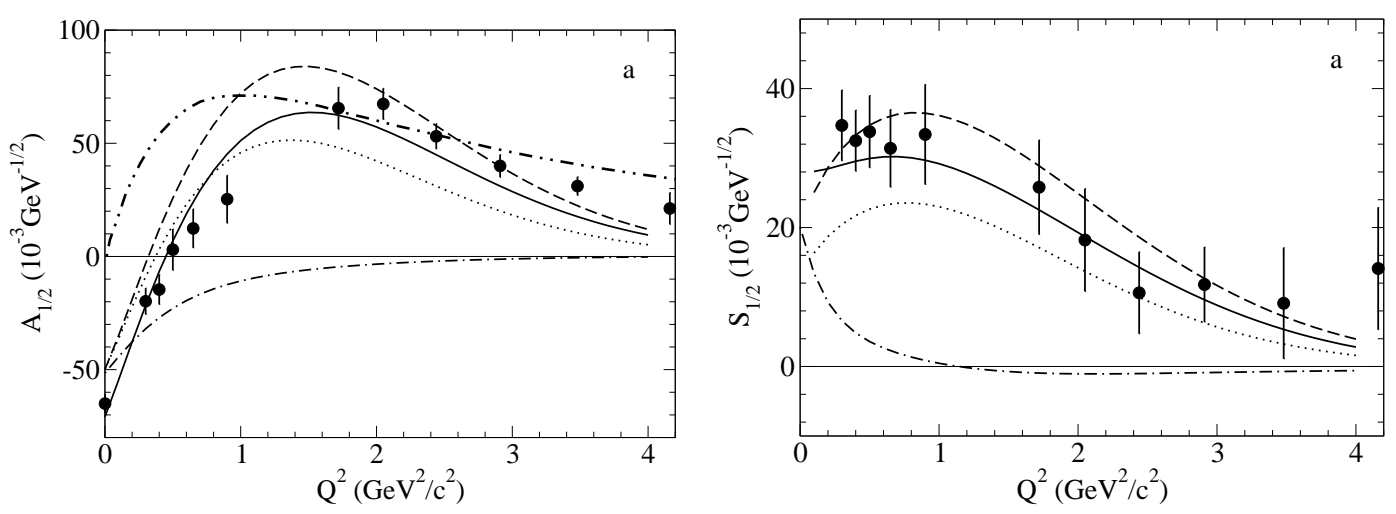

Figure 3: Helicity amplitudes $A_{1 / 2}$ and $S_{1 / 2}$ in comparison to JLab data [曰. 5 . Dotted curves — the quark core excitation amplitudes $|3 q\rangle+\gamma^{*} \rightarrow\left|3 q^{*}\right\rangle$ calculated in the framework of the standard ${ }^{3} P_{0}+$ VMD' model with a fixed vector meson radius $b_{V}=y_{0} b$. Dashed curves - the same amplitudes calculated in a modified ${ }^{3} P_{0}+$ VMD' model with a $Q^{2}$-dependent scale parameter $y=y_{0} e^{-Q^{2} / \chi^{2}}$ for the vector meson radius $b_{V}=y b$. Dashed-dotted curves - helicity amplitudes for the electroexcitation of the hadron molecule $N+\sigma$. Solid curves - the full calculation of $A_{1 / 2}$ and $S_{1 / 2}$ in terms of a combined structure $R=\cos \theta\left|3 q^{*}\right\rangle+\sin \theta|N+\sigma\rangle$. For comparison, the valence quark contribution to $A_{1 / 2}$ calculated in Ref. [8] on the basis of a covariant spectator model is also shown (the dashed-double-dotted curve in the left top panel).

For the $\sigma$ resonance we take values which are reasonable [1] (a wide range of values is given by $M_{\sigma}=(0.4-1.2) \mathrm{GeV}, \Gamma_{\sigma}=(0.5-1) \mathrm{GeV}$ and $\left.g_{\sigma_{N N}} \approx 5-10\right)$. Some fine-tuning of these param- 


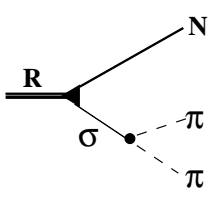

Figure 4: $R \rightarrow N+(\pi+\pi)_{I=0}$ decay process via the $\sigma$-meson resonance.

eters to the complete range of data on $A_{1 / 2}$ results in the following set of molecular parameters: $\Lambda_{M}=1 \mathrm{GeV}, \Lambda_{N}=0.8 \mathrm{GeV}, M_{\sigma}=0.5 \pm 0.05 \mathrm{GeV}, \Gamma_{\sigma}=0.75 \pm 0.25 \mathrm{GeV}, g_{\sigma_{N N}}=5$. The mixing parameter $\theta$ is fixed in the low energy region $\left(0 \lesssim Q^{2} \lesssim 1 \mathrm{GeV}^{2} / c^{2}\right)$ of $A_{1 / 2}$, where the molecular component is optimized to reproduce the differnece between the $3 q$ contribution and the JLab data.

The calculated helicity amplitudes $A_{1 / 2}$ and $S_{1 / 2}$ are shown in Figs. . We also show separately the contributions to the amplitude from the quark and the hadron molecule components (dashed and dashed-dotted curves, respectively). The comparison with the standard ${ }^{3} P_{0}$ model calculation with a fixed value for the vector-meson radius $b_{V}=0.9 b$ (dotted curves) demonstrates the following: a smooth transition from the ${ }^{3} P_{0} \gamma R N$ vertex (Fig. 1 $1 \mathrm{a}$ ) to the parton-like one (Fig. 1 $1 \mathrm{~b}$ ) using a $Q^{2}$ dependent vector meson radius $b_{V}\left(Q^{2}\right) \rightarrow 0$ leads to considerable improvement of the standard ${ }^{3} P_{0}$ model results at moderate values of $Q^{2}$.

The quark core component of $\mathrm{R}$ plays the main role in the electroproduction of the Roper resonance for this $Q^{2}$ region $\left(Q^{2} \gtrsim 1-1.5 \mathrm{GeV}^{2} / c^{2}\right)$. For small values of $Q^{2} \lesssim 1 \mathrm{GeV}^{2}$, where the contribution of the meson cloud should also be important, it can be effectively taken into account in the framework of ${ }^{3} P_{0}$ - and VMD models. However, such a model overestimates the transverse amplitude $A_{1 / 2}$ in the region $0.5 \lesssim Q^{2} \lesssim 1 \mathrm{GeV}^{2}$ (the dashed line in Fig. B). The description of the JLab data [4. 可 on $A_{1 / 2}$ can be considerably improved if one takes a combined structure for the Roper in the form of $|R\rangle=\cos \theta\left|3 q^{*}\right\rangle+\sin \theta|N+\sigma\rangle$. The adjustable parameter $\theta$ fitted to the JLab data in this region is $\cos \theta=0.8$ indicating an admixture of $N \sigma$ component of about $36 \%$.

When the weight of $N+\sigma$ component in the Roper resonance in terms of $\sim \sin ^{2} \theta$ is fixed, the Roper decay width for the transition $N+(\pi \pi)_{\text {Swave }}^{I=0}$ can be calculated. The assumption that the quark part of the Roper just gives a very small contribution through a virtual transition $R \rightarrow N+\sigma$ is justified in our quark model. Then the transition is described as the virtual decay of the molecular part to $N+\sigma$ followed by the $\sigma \rightarrow \pi \pi$ decay. The diagram for such a mechanism is shown in Fig. $\theta$. The probability $\left|M_{f i}\right|^{2}$ for the transition process of Fig. 4 contains the Breit-Wigner representation for the intermediate $\sigma$-meson state with

$$
\left|M_{f i}\right|^{2}=g_{R \sigma N}^{2} g_{\sigma \pi \pi}^{2} \tilde{\Phi}_{R}^{2}\left(k^{2}\right) \frac{\left(m_{N}+m_{R}\right)^{2}-s_{\pi \pi}}{\left(m_{\sigma}^{2}-s_{\pi \pi}\right)^{2}+m_{\sigma}^{2} \Gamma_{\sigma}^{2}\left(s_{\pi \pi}\right)}, \Gamma_{\sigma}(x)=\Gamma_{\sigma} \frac{m_{\sigma}}{\sqrt{x}} \frac{\sqrt{x-4 m_{\pi}^{2}}}{\sqrt{m_{\sigma}^{2}-4 m_{\pi}^{2}}},
$$

where $k=p_{R}-\omega_{N \sigma} p_{N}, x=s_{\pi \pi} \equiv k_{\sigma}^{2}$ and the coupling constant $g_{\sigma \pi \pi}$ is deduced from the twopion decay width of the $\sigma$ with $g_{\sigma \pi \pi}^{2}=\frac{32 \pi}{3} \Gamma_{\sigma} m_{\sigma}\left(1-\frac{4 m_{\pi}^{2}}{m_{\sigma}^{2}}\right)^{-1 / 2}$. The coupling constant $g_{R \sigma N}$ of the hadron-molecular vertex is defined by the compositeness condition (2.6). The numerical value for $\Gamma_{R \rightarrow N \sigma}$ with $g_{R \sigma N}=6.39$ [fixed by the compositeness condition (2.6)] and a molecular admixture in the Roper of $\sin \theta \simeq 0.6$ is $\Gamma_{R \rightarrow N \sigma(\pi \pi)}=(19.0-26.7) \mathrm{MeV}$, where the lower and upper limits correspond to a variation of the $\sigma$ decay width $\Gamma_{\sigma}$ from 0.5 to $1 \mathrm{GeV}$, respectively (the variation of the $\sigma$-meson mass $M_{\sigma}=500 \pm 50 \mathrm{MeV}$ can only change the result within $10 \%$ ). This should 
be compared to the PDG [1] value $\Gamma_{R \rightarrow N \sigma(\pi \pi)} \approx(0.05-0.1) \Gamma_{R}^{\text {tot }}(\approx 15-30 \mathrm{MeV})$ or the recent data [2] $\Gamma_{R \rightarrow N \sigma}=71 \pm 17 \mathrm{MeV}$. It is clear that the strong Roper decay can serve as a constraint on $\Gamma_{\sigma}$, however present results for $\Gamma_{R \rightarrow N \sigma(\pi \pi)}$ are compatible with all values of $\Gamma_{\sigma}$.

The pion decay width calculated for the quark part of the Roper resonance in the framework of our approach $\left(\Gamma_{R \rightarrow \pi N}^{q} \simeq 36 \mathrm{MeV}\right)$ is not as small as in the case of EEM evaluations $\left(\Gamma_{R \rightarrow \pi N}^{E E M} \simeq 4\right.$ $\mathrm{MeV})$ but it is still several times smaller than the PDG value of $\Gamma_{R \rightarrow N+\pi} \approx(0.55-0.75) \Gamma_{R}^{\text {tot }}$. It is clear that considerable corrections to $\Gamma_{R \rightarrow \pi N}^{q}$ can come from the pion cloud contribution which is neglected here.

\section{Conclusions}

We suggested a two-component model of the lightest nucleon resonance $R=N_{1 / 2^{+}}(1440)$ as a combined state of the quark configuration $s p^{2}[3]_{X}$ and the hadron molecule component $N+\sigma$. This approach allows to describe with reasonable accuracy the recent CLAS electroproduction data [4. 5] at low- and moderate values of $Q^{2}$ with $0 \leq Q^{2} \lesssim 1.5-2 \mathrm{GeV}^{2}$. In the model the $R \rightarrow N+(\pi \pi)_{\text {Swave }}^{I=0}$ transition process is interpreted as the decay of a virtual $\sigma$ meson in the $N+\sigma$ component. The calculated decay width $\Gamma_{R \rightarrow N \sigma(\pi \pi)}$ correlates well with the PDG value [1] and the recent CB-ELSA and A2-TAPS data [2].

We tried to show that the description of transition amplitudes in terms of parton-like models, which are very good at high $Q^{2}$, can be naturally transformed into a description in terms of the "soft" vector meson cloud. This smooth transition is achieved by 'switching on' a non-zero radius of the intermediate vector meson. The vector meson $V$ of finite size generates a non-local $V q q$ interaction. This weakens the effect of the orthogonality of the spatial $R$ and $N$ wave functions in the transition matrix element $N+\gamma_{T}^{*} \rightarrow R$, and the amplitude $A_{1 / 2}$. Resulting theoretical values, which match the data, are contrary to the standard predictions of LF-models, which lead to non-zero and (negative) large values at the photon point.

Further we plan to develop a relativistic version of the suggested electroexcitation mechanism.

\section{References}

[1] K. Nakamura et al. (Particle Data Group), J. Phys. G 37, 075021 (2010).

[2] A. V. Sarantsev et al. (CB-ELSA and A2-TAPS collaborations), Phys. Lett. B 659, 94 (2008).

[3] N.Suzuki et al., Phys. Rev. Lett, 104, 042302 (2010); arXiv:0909.1356[nucl-th]

[4] I. G. Aznauryan et al. (CLAS Collaboration), Phys. Rev. C 80, 055203 (2009).

[5] V. I. Mokeev, V. D. Burkert, T. S. Lee, L. Elouadrhiri, G. V. Fedotov and B. S. Ishkhanov (CLAS collaboration), Phys. Rev. C 80, 045212 (2009); V.I. Mokeev, arXiv:1010.0712[nucl-ex];

G.V.Fedotov, et al., CLAS Collaboration, Phys. Rev. C79, 015204 (2009).

[6] V. D. Burkert and T. S. H. Lee, Int. J. Mod. Phys. E 13, 1035 (2004).

[7] S. Capstick, B. D. Keister and D. Morel, J. Phys. Conf. Ser. 69, 012016 (2007).

[8] G. Ramalho and K. Tsushima, Phys. Rev. D 81, 074020 (2010).

[9] B. Golli, S. Sirca and M. Fiolhais, Eur. Phys. J. A 42, 185 (2009). 
[10] A. Matsuyama, T. Sato and T. S. Lee, Phys. Rept. 439, 193 (2007).

[11] N. Suzuki, T. Sato, and T. S. Lee, Phys. Rev. C 76, 025205 (2007); B. Julia-Diaz, T. S. Lee, A. Matsuyama and T. Sato, Phys. Rev. C 76, 065201 (2007); J. Liu, R. D. McKeown and M. J. Ramsey-Musolf, Phys. Rev. C 76, 025202 (2007).

[12] G. V. Fedotov et al. (CLAS Collaboration), Phys. Rev. C 79, 015204 (2009)

[13] I. G. Aznauryan, V. D. Burkert, G V. Fedotov, B. S. Ishkhanov, and V. I. Mokeev, Phys. Rev. C 72, 045201 (2005).

[14] V. I. Mokeev and V. D. Burkert, J. Phys. Conf. Ser. 69, 012019 (2007).

[15] B. Borasoy, P. C. Bruns, U. G. Meissner and R. Lewis, Phys. Lett. B 641, 294 (2006). [arXiv:hep-lat/0608001].

[16] D. Djukanovic, J. Gegelia and S. Scherer, Phys. Lett. B 690, 123 (2010).

[17] I. G. Aznauryan, Phys. Rev. C 76, 025212 (2007).

[18] D. Robson, Nucl. Phys. A 560, 389 (1993); U. Meyer, A. J. Buchmann and A. Faessler, Phys. Lett. B 408, 19 (1997).

[19] F. Cano and P. Gonzalez, Phys. Lett. B 431, 270 (1998).

[20] M.B. Gavella, A. Le Yaouanc, L. Oliver et al, Phys. Rev. D21, 182 (1980).

[21] I.T. Obukhovsky, A. Faessler, D.K. Fedorov, Th. Gutsche, and V.E. Lyubovitskij, Phys. Rev. D84, 014004 (2011).

[22] F. Gross, G. Ramalho and M. T. Pena, Phys. Rev. C 77, 015202 (2008); F. Gross, G. Ramalho and K. Tsushima, Phys. Lett. B 690, 183 (2010).

[23] O. Krehl, C. Hanhart, S. Krewald, J. Speth, Phys. Rev. C62 , 025207 (2000); arXiv:nucl-th/9911080.

[24] E. S. Ackleh, T. Barnes and E. S. Swanson, Phys. Rev. D 54, 6811 (1996).

[25] A. Faessler, T. Gutsche, V. E. Lyubovitskij, Y. L. Ma, Phys. Rev. D 76, 014005 (2007); Phys. Rev. D 80, 054019 (2009); Phys. Rev. D 80, 054019 (2009); Y. Dong, A. Faessler, T. Gutsche, S. Kumano and V. E. Lyubovitskij, Phys. Rev. D 82, 034035 (2010).

[26] Yu. S. Kalashnikova, Phys. Rev. D 72, 034010 (2005).

[27] T. Barnes, F.E. Close, P.R. Page, and E.S. Swanson, Phys. Rev. D 55, 4157 (1997); T. Barnes, N. Black, and P.R. Page, Phys. Rev. D 68, 054014 (2003); T. Barnes, S. Godfray, and E.S. Swanson, Phys. Rev. D 72, 054026 (2005).

[28] C.B. Dover, T. Gutsche, M. Maruyama, and Amand Faessler, Prog. Part. Phys. 29, 87 (1992).

[29] S. Weinberg, Phys. Rev. 130, 776 (1963); A. Salam, Nuovo Cim. 25, 224 (1962).

[30] G. V. Efimov and M. A. Ivanov, The Quark Confinement Model of Hadrons, (IOP Publishing, Bristol \& Philadelphia, 1993).

[31] I. V. Anikin, M. A. Ivanov, N. B. Kulimanova and V. E. Lyubovitskij, Z. Phys. C 65, 681 (1995); M. A. Ivanov, M. P. Locher and V. E. Lyubovitskij, Few Body Syst. 21, 131 (1996) M. A. Ivanov, V. E. Lyubovitskij, J. G. Körner and P. Kroll, Phys. Rev. D 56, 348 (1997); A. Faessler, T. Gutsche, M. A. Ivanov, J. G. Korner and V. E. Lyubovitskij, Phys. Rev. D 80, 034025 (2009).

[32] S. Capstick and B. D. Keister, Phys. Rev. D 51, 3598 (1995).

[33] S. Mandelstam, Annals Phys. 19, 1 (1962).

[34] J. Terning, Phys. Rev. D 44, 887 (1991). 